\title{
Analytical solution for temporal photorefractive response in the diffusion regime
}

\author{
Marek Wichtowski and Ewa Weinert-Rączka* \\ Faculty of Electrical Engineering, West Pomeranian University of Technology, al Piastów 17, 70-310 Szczecin
}

Received December 14, 2009; accepted December 30, 2009; published December 31, 2009

\begin{abstract}
The evolution of a space-charge field during photorefractive two wave mixing in unbiased sample is considered. An approximate analytical solution describing the photorefractive material response to the interference pattern of arbitrary fringe contrast is presented. Obtained solutions are compared with the results of numerical simulations.
\end{abstract}

The photorefractive (PR) effect relies on the refractive index changes induced by non-uniform illumination in the photoconductive and electro-optic material. The PR effect is commonly explained in the framework of the band transport model [1], which assumes photogeneration of free carriers, their transport and trapping in dark regions. The redistribution of carriers generates the space-charge electric field $\left(\mathrm{E}_{\mathrm{sc}}\right)$ inside the crystal. The material equations of this model are nonlinear and coupled, therefore they do not have an exact analytical solution in a general case. One of the most often investigated geometries is the two-wave mixing (TWM) configuration where interfering waves create a sinusoidal distribution of light intensity. Several methods of the space-charge field determination in such a case were developed. The majority of research is devoted to the study of steady state solutions. The dynamics of PR grating is essentially described in the frames of two approaches. For a small contrast of interference fringes, a linearization of material equations can be made. A high fringe contrast case is usually studied by means of numerical methods. To date very few attempts to find an analytical solution for the transient response of a photorefractive crystal have been taken. For example, an approximate analytical solution for space-charge field evolution given in [2] is valid only for the grating period much larger than the Debye length. In our paper we present an alternative time-dependent analytical solution applicable for arbitrary modulation depth of the interference pattern. The relationship that describes directly the grating formation dynamics in TWM geometry without external electric field was found. The applied approach is based on the idea presented in the work [3].

\footnotetext{
"E-mail: ewa.raczka@ps.pl
}

We consider the band transport model with one type of charge carriers (electrons), and one photoactive donor level of dopants with concentration $N_{\mathrm{D}}$ which are partially compensated by shallow fully ionized acceptors with concentration $N_{\mathrm{A}}=N_{\mathrm{A}}{ }^{-}=$constant. In the considered case no external electric field is applied to the crystal, so the electron transport is caused only by diffusion. A photorefractive transport model is described by the following set of equations:

$$
\begin{gathered}
\partial N_{\mathrm{D}}^{+} / \partial t=S I\left(N_{\mathrm{D}}-N_{\mathrm{D}}^{+}\right)-\gamma N_{\mathrm{D}}^{+} n, \\
\partial \rho / \partial t=-\partial j / \partial z, \\
j=q \mu n E_{\mathrm{sc}}+\mu k_{\mathrm{B}} T \partial n / \partial z \\
\partial E_{\mathrm{sc}} / \partial z=\rho / \varepsilon
\end{gathered}
$$

where $n, N_{\mathrm{D}}, N_{\mathrm{D}}^{+}, N_{\mathrm{A}}$ are electron, donor, ionized donor and acceptor densities, respectively. $S$ describes photoexcitation cross section, $I$ - light intensity, $\gamma$ recombination constant, $j$ - current density, $\mu$ - electron mobility $k_{\mathrm{B}}-$ Boltzmann's constant, $T$ - absolute temperature, $\varepsilon=\varepsilon_{0} \varepsilon_{\mathrm{r}}$ an electric permittivity and $\rho=q\left(N_{\mathrm{D}}^{+}-n-N_{\mathrm{A}}^{-}\right)$charge density. Additionally, we have the external condition:

$$
\int_{0}^{L} E_{s c}(z) d z=0
$$

The photorefractive response is induced by two plane waves creating an interference pattern of the form

$$
I(z, t)=I_{0}[1+m \exp (i K z)+\text { c.c. }] \theta(t),
$$

where $I_{0}=I_{1}+I_{2}$ is the average intensity, $m-$ the modulation depth, $K=2 \pi / \Lambda$ - the grating constant, and $\theta(t)$ - Heaviside's step function.

For the crystal length much bigger than the grating period $\Lambda$ the periodic boundary conditions can be applied. In this case the condition (3) can be replaced by averaging over one grating period and all variables $\left(\mathrm{N}_{\mathrm{D}}^{+}\right.$, $\left.n, E_{\mathrm{sc}}\right)$ can be expanded in the Fourier series:

$$
V(z, t)=V_{0}(t)+\sum_{n=1}^{\infty} V_{n}(t) e^{i n K z}+\text { c.c. } \approx \sum_{n=-N}^{N} V_{n}(t) e^{i n K z}
$$


where $V_{0}$ represents average values, and $V_{\mathrm{n}}(t)=\left|V_{\mathrm{n}}(t)\right| \mathrm{e}^{\mathrm{i} \phi n}$ are complex amplitudes, $\phi_{n}$ reflects the fact that the variables distributions can exhibit spatial shifts in respect to the interference pattern.

Substituting extensions (6) (for $N$ components) to the set of equations (1a), (1d) and comparing the terms with the same exponential factor $\exp (\mathrm{i} n K z)$, one obtains equations for the amplitudes of successive harmonics. The form of these equations can be found in [2] Numerical simulations carried out in our paper have been done using this system of equations.

Characteristic temporal scales for evolution of space charge distributions can be determined by assuming small fringes contrast $m<<1$ and making linearization of material equations. For this purpose we substitute variables (4) limited to the first order terms to the equations (1), obtaining for zero order components $N_{\mathrm{D} 0}{ }^{+}(t) \cong N_{\mathrm{A}}$ (which arises from the fact that $n<<$ $N_{\mathrm{D}}{ }^{+}, N_{\mathrm{D}}{ }^{0}$ ) and an additional equation

$$
\mathrm{d} n_{0}(t) / \mathrm{d} t=\operatorname{SI}(t)\left(N_{D}-N_{A}\right)-n_{0}(t) / \tau_{\mathrm{e}} .
$$

As can be seen, the average electron concentration attains the steady state value $n_{0}=S I_{0}\left(N_{D}-N_{A}\right) \tau_{\mathrm{e}}$ in the time-scale determined by the carrier recombination time $\tau_{\mathrm{e}}=1 /\left(\gamma N_{A}\right)$. This time is much shorter than the space charge formation time, which means that in the case of abrupt light onswitching, one can assume $n_{0}(t) \approx n_{0}$.

For the first order amplitudes the set of equations takes the form:

$$
\frac{d}{d t}\left(\begin{array}{c}
n_{1} \\
N_{D 1}^{+}
\end{array}\right)=\left(\begin{array}{cc}
-\tau_{e}^{-1}-\mu K E_{d} & -\tau_{0}^{-1}+\tau_{d i e}^{-1} \\
-\tau_{e}^{-1} & -\tau_{0}^{-1}
\end{array}\right)\left(\begin{array}{c}
n_{1} \\
N_{D 1}^{+}
\end{array}\right)+B\left(\begin{array}{l}
1 \\
1
\end{array}\right)
$$

where $B=m S I_{0}\left(N_{D}-N_{A}\right), E_{\mathrm{d}}=\left(k_{\mathrm{B}} T / q\right) K$ is called the diffusion field and $\tau_{\mathrm{die}}=\varepsilon / \sigma_{0}=\varepsilon /\left(q \mu n_{0}\right)$ is the dielectric relaxation time, which depends on the mean photoconductivity $\sigma_{0}$ and the average life-time of ionized traps $\tau_{0}=1 /\left(m_{0}\right)$.. The space-charge field amplitude results from Gauss law (1d)

$$
E_{\mathrm{sc}}(t) \approx E_{1}(t)=(-\mathrm{i} q / \varepsilon K) N_{\mathrm{D} 1}^{+}(t) .
$$

To solve (6.1) one should note that free electron distribution $n_{1}(z, t)$ attains the quasi-equilibrium state with the space charge distribution $N_{\mathrm{D}}{ }^{+}(z, t)$ in very short time (of the order of $\tau_{\mathrm{e}}$ ) and follows the space charge changes with a negligible delay. This approximation allows to set $\mathrm{d} n_{1} / \mathrm{d} t=0$ in the first equation of (6a) and to obtain a solution of the set $(6 a)-(6 b)$ in the form:

$$
V_{1}(t)=V_{1}(\infty)[1-\exp (-t / \tau)]
$$

Time constant describing the dynamics of the space charge grating formation is given by

$$
\tau=\tau_{\mathrm{die}}\left(1+K^{2} L_{\mathrm{D}}^{2}\right) /\left(1+K^{2} L_{\mathrm{Db}}{ }^{2}\right),
$$

where $L_{\mathrm{D}}$ i $L_{\mathrm{Db}}$ denote respectively: the diffusion length given by $K^{2} L_{\mathrm{D}}{ }^{2}=\mu \tau E_{\mathrm{d}}$, and the Debye screening length given by $K^{2} L_{\mathrm{Db}}{ }^{2}=E_{\mathrm{d}} / E_{\mathrm{q}}$, with $E_{\mathrm{q}}=(q / \varepsilon K)\left(N_{\mathrm{D}}-N_{\mathrm{A}}\right) N_{\mathrm{A}} / N_{\mathrm{D}}$ called the saturation field. The amplitude of the $E_{\mathrm{sc}}$ field in a steady state is

$$
E_{1}(\infty)=-i m_{1} E_{\mathrm{d}} ; \quad m_{1}=m /\left(1+K^{2} L_{\mathrm{Db}}^{2}\right),
$$

while the amplitude of electron density is $n_{1}(\infty)=m_{1} n_{0}$.

To find a solution for arbitrary fringe contrast we employ the continuity equation (1b):

$$
\partial \rho(z, t) / \partial t=-\partial j(z, t) / \partial z
$$

Determining the charge density from Gauss's law (1d), substituting into (1b) and integrating over $z$ we obtain

$$
\varepsilon \cdot \partial E_{\mathrm{sc}}(z, t) / \partial t=-j(z, t)+j_{0}(t) .
$$

The integration constant $j_{0}(t)$ can be found by taking into account the condition $\left\langle E_{\mathrm{sc}}\right\rangle=0$ and spatially averaging both sides of equation (8b), which results in $j_{0}(t)=\langle j(z, t)\rangle$. In the diffusion transport case an average current is equal to zero, hence $j_{0}(t)=0$.

In order to find an expression for $j(z, t)$ we assume that in a transient state the profile of free carrier density remains quasi-sinusoidal: $n(z, t)=n_{0}+n_{1}(t) \cos (K z)$. Denoting conductivities of zero and first order by $\sigma_{0}=$ $q \mu n_{0}$ and $\sigma_{1}(t)=q \mu n_{1}(t)$, equation (1c) takes the form:

$j(z, t)=\left[\sigma_{0}+\sigma_{1}(t) \cdot \cos (K z)\right] \cdot E_{\mathrm{sc}}(z, t)-\sigma_{1}(t) E_{\mathrm{d}} \cdot \sin (K z)$.

Substituting (9) into equation (8b) one obtains

$\varepsilon \cdot \partial E_{\mathrm{sc}} / \partial t+\left[\sigma_{0}+\sigma_{1}(t) \cos (K z)\right] \cdot E_{\mathrm{sc}}+\sigma_{1}(t) \cdot E_{\mathrm{d}} \sin (K z)=0$.

From (10) one can easily find the spatial distribution of $E_{\mathrm{sc}}$ for the steady state. Taking into account that $\sigma_{1}(\infty)=$ $m_{1} \sigma_{0}$, where $m_{1}$ is given by $(7 \mathrm{c})$ we get $E_{\mathrm{sc}}(z)$ and its Fourier components:

$$
E_{s c}(z)=\frac{m_{1} E_{d} \sin (K z)}{1+m_{1} \cos (K z)} ; \quad E_{n}=2 i\left(\frac{\sqrt{1-m_{1}^{2}}-1}{m_{1}}\right)^{n} E_{d}
$$

In equation (10) the dependence $\sigma_{1}(t)$ is unknown. We assume that $\sigma_{1}(t)$ is approximately given by the same expression as linear solutions in $(7 \mathrm{a})$ :

$$
\sigma_{1}(t)=m_{1} \sigma_{0} \cdot[1-\exp (-t / \tau)] .
$$

In this case, for the transient process under the initial condition $E_{s c}(z, 0)=0$, the solution of $(10)$ is in the form

$$
E_{s c}(z, t)=m_{1} E_{d} \sin (K z) \int_{0}^{t}\left(1-e^{-t / \tau}\right) e^{\Phi\left(t, t^{\prime}\right)} d t^{\prime}
$$




$$
\Phi\left(t, t^{\prime}\right)=\frac{t^{\prime}-t}{\tau_{\text {die }}}+\varepsilon^{-1} \cos (K z)\left(\int_{0}^{t^{\prime}} \sigma_{1}(\eta) d \eta-\int_{0}^{t} \sigma_{1}(\eta) d \eta\right) .
$$

The dependence (12) enables one to determine the evolution of the space charge field profile, which is presented in Fig.1.

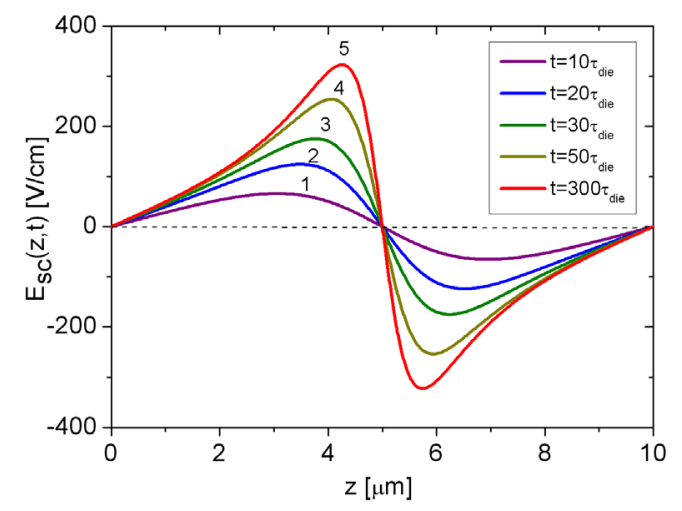

Fig.1. Space charge field distribution $E_{\mathrm{sc}}(\mathrm{z}, \mathrm{t})$ calculated from (12), for few values of $t\left(10 \tau_{\text {die }}, 20 \tau_{\text {die }}, 30 \tau_{\text {die }}, 50 \tau_{\text {die }}\right.$ and $\left.300 \tau_{\text {die }}\right)$.

Harmonics amplitudes of $\mathrm{E}_{\mathrm{sc}}(\mathrm{z})$ can be found using the expansions:

$$
E_{s c}(z, t)=\sum_{n=-\infty}^{\infty} E_{n} e^{i n K z}+c . c .
$$

and

$$
e^{\xi \cos (K z)}=\sum_{n=-\infty}^{\infty} I_{n}(\xi) e^{i n K z}
$$

where $I_{n}(\xi)$ denote the modified Bessel functions of the first kind and $n$-th order. As a result, one gets complex amplitudes of the space-charge field:

$$
\begin{gathered}
E_{n}(t)=2 i H_{n}(t) E_{\mathrm{d}} \\
H_{n}=-\frac{m_{1}}{2} \int_{0}^{t}\left(1-e^{-t^{\prime} / \tau_{n}}\right) e^{\left(t^{\prime}-t\right) / \tau_{d i e}}\left(I_{n+1}(X)-I_{n-1}(X)\right) d t^{\prime} \\
X=m_{1}\left(\left(t^{\prime}-t\right) / \tau_{d i e}+\left(e^{-t^{\prime} / \tau_{n}}-e^{-t / \tau_{n}}\right) \tau_{n} / \tau_{d i e}\right) ; \tau_{n}=n^{2} \cdot \tau
\end{gathered}
$$

For $t \rightarrow \infty$ function $H_{n}(t)$ tends to a value given by (11). The testing of $H_{1}(t)$ reveals that increasing fringe contrast increases the recording time of the grating, which is in agreement with experimental observations [4]. The time evolution of the first three harmonic amplitudes of $E_{\mathrm{sc}}$ for the modulation depth $m=0.9$ and material parameters listed in Table 1 calculated from (14) are presented in Fig.2. To asses the accuracy of expression (14) the solution was compared with the results of numerical simulations. In both methods the harmonics of higher order show slower growth rates and emerge with initial delay times in respect to the fundamental harmonic.

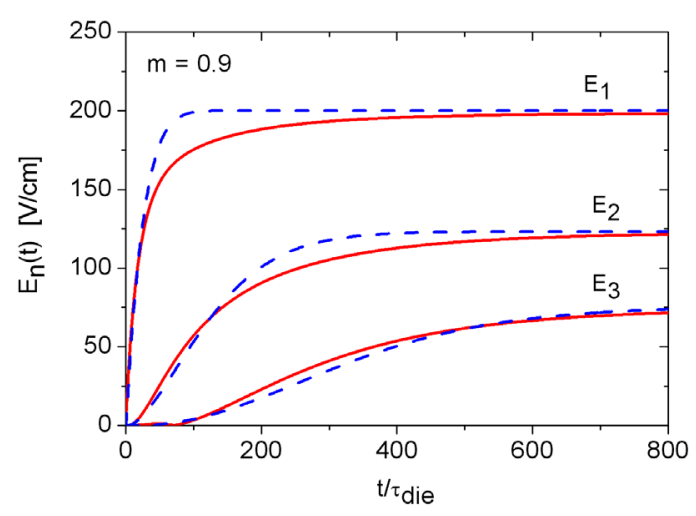

Fig.2. Time dependence of the first three harmonic components of $E_{\mathrm{sc}}$ in the case of diffusion transport. Solid lines - the numerical solution, dashed lines - the analytical solution given by (14). The modulation depth is $m=0.9$, the grating period is $\Lambda=10 \mu \mathrm{m}$, the dielectric relaxation time $\tau_{\mathrm{die}} \approx 1 \mu \mathrm{s}$.

\section{TABle 1. MATERIAL PARAMETERS FOR GAAs:CR}

\begin{tabular}{ll}
\hline Deep donor concentration & $N_{\mathrm{D}}=1 \times 10^{16} \mathrm{~cm}^{-3}$ \\
Acceptor concentration & $N_{\mathrm{A}}=1 \times 10^{15} \mathrm{~cm}^{-3}$ \\
Electron mobility & $\mu=6000 \mathrm{~cm}^{2} / \mathrm{Vs}$ \\
Cross section of photoionization & $s=10^{-16} \mathrm{~cm}^{2} ; S=s / h v$ \\
Recombination constant & $\gamma=4 \times 10^{-7} \mathrm{~cm}^{3} / \mathrm{s}$ \\
Relative dielectric constant & $\varepsilon_{\mathrm{r}}=12.3$ \\
Wavelength & $\lambda=1.06 \mu \mathrm{m}$ \\
Grating period & $\Lambda=10 \mu \mathrm{m}$ \\
Average light intensity & $I_{0}=100 \mathrm{~mW} / \mathrm{cm}^{2}$ \\
\hline
\end{tabular}

The approximate analytical solutions presented here permit to carry out fast simulation of temporal behavior of the photorefractive grating in a TWM experiment performed in a diffusion regime. The solutions exhibit features confirmed by experimental observations. Comparison with the numerical results confirms the validity of the approximate solutions at high modulation depth.

\section{References}

[1] J.P. Huignard, P. Gunter, Photorefractive Materials and Their Applications I (Springer-Verlag, Berlin, 1988)

[2] E. Serrano, M. Carrascosa, F. Agulló-López, JOSA B 13. 2587 (1996).

[3] B.I.Sturman, E. F. Agulló-López, M. Carrascosa, L.Solymar, Appl. Phys. B 68, 1013 (1999)

[4] J.E. Millerd, E.M.Garmire, M.B. Klein, B.A.Wechsler, E.P.Strohkendl, G.A. Brost, JOSA B 9, 1449 (1992) 